\title{
Kinetics of Nonisothermal Degradation of Some Polymer Composites: Change of Entropy at the Formation of the Activated Complex from the Reagents
}

\author{
Sevdalina Turmanova, ${ }^{1}$ Svetlana Genieva, ${ }^{2}$ and Lyubomir Vlaev ${ }^{3}$ \\ ${ }^{1}$ Department of Material Science, Assen Zlatarov University, 8010 Burgas, Bulgaria \\ ${ }^{2}$ Departments of Inorganic and Analytical Chemistry, Assen Zlatarov University, 8010 Burgas, Bulgaria \\ ${ }^{3}$ Department of Physical Chemistry, Assen Zlatarov University, 8010 Burgas, Bulgaria
}

Correspondence should be addressed to Sevdalina Turmanova, sturmanova@btu.bg

Received 21 November 2010; Revised 24 January 2011; Accepted 21 March 2011

Academic Editor: Krzysztof J. Ptasinski

Copyright (C) 2011 Sevdalina Turmanova et al. This is an open access article distributed under the Creative Commons Attribution License, which permits unrestricted use, distribution, and reproduction in any medium, provided the original work is properly cited.

\begin{abstract}
Studying the nonisothermal kinetics of degradation of rice husks in air or nitrogen atmosphere, polypropylene and tetrafluoroethylene-ethylene copolymer filled with different quantities of rice husks flour or the products of its thermal degradation, namely "white" or "black" rice husks ash, a linear dependence was observed between the $\ln A$ and $E_{A}$, known as the kinetic compensation effect or theta rule. A linear relationship was also established between $E_{A}$ and the change of the entropy $\Delta S^{\neq}$for the formation of the activated complex from the reagents. These dependences are related to the assumption of identical kinetic mechanisms of thermal degradation of the composites studied. The negative values of $\Delta S^{\neq}$obtained show that the activated complex is a "more organized" structure than the initial reactants and that these reactions may be classified as "slow" ones. It may be concluded that the products of the thermal degradation of rice husks in a fluidized bed reactor can successfully replace the more expensive synthetic fillers to obtain different polymer composites. These polymer composites can lead to the futuristic "organic-inorganic hybrid materials" with specific properties.
\end{abstract}

\section{Introduction}

It is well known that the nonisothermal thermogravimetric analysis may successfully be used for determination of the kinetic triplet: the apparent activation $E_{A}$, the pre-exponential factor $A$ in the Arrhenius equation, and the shape of the most probable mechanism function $f(\alpha)$ for some heterogeneous reactions [1-4]. If the kinetic studies are carried out on similar compounds and with a correctly chosen mechanism function $f(\alpha)$, a linear relationship between the logarithm of the pre-exponential factors and activation energies exists, known as the kinetic compensation effect (KCE), isokinetic effect, or theta rule [5-8]. Such a correlation was firstly reported in 1925 by Constable [9], in connection with his catalytic studies. Several theories and explanations accounting for such compensation behavior have also been put forward [9-12]. Evidence of such a compensation effect has since been reported in many publications [13-17], even though some of these reports have subsequently been disputed [18-21].

In the case of studies of thermal decomposition reactions of solids, the existence of the compensation effect allows of certain conclusions concerning the decomposition mechanism and thermal characteristics of the compounds under investigation. Studying the kinetics of the thermal decomposition of different solid compounds at nonisothermal heating, for instance, using the TG curves recorded, a proper calculation procedure, and the algebraic expression of the conversion function $f(\alpha)$, one can calculate not only the values of the activation energy and frequency factor in the Arrhenius equation but also the changes of the Gibbs energy $\Delta G^{\neq}$, enthalpy $\Delta H^{\neq}$, and entropy $\Delta S^{\neq}$for the 
formation of the activated complex from the reagents, respectively. The change of the Gibbs energy $\Delta G^{\neq}$reflects the total energy increase of the system at the approach of the reagents and the formation of the activated complex. This energy is influenced by two thermodynamic parameters: the changes of enthalpy $\Delta H^{\neq}$and entropy $\Delta S^{\neq}$for the formation of the activated complex. The change of the activation enthalpy shows the energy differences between the activated complex and the reagents. If this difference is small, the formation of the activated complex is favored since the potential energy barrier is low. The change of the activation entropy $\Delta S^{\neq}$reflects how near the system is to its own thermodynamic equilibrium. Low activation entropy means that the material has just passed through some kind of physical or chemical aging process, bringing it to a state near its own thermodynamic equilibrium. In this situation, the material shows little reactivity, increasing the time taken to form the activated complex. On the other hand, when high activation entropy values are observed, the material is far from its own thermodynamic equilibrium. In this case, the reactivity is high, and the system can react faster to produce the activated complex, which gives shorter observed reaction times [22,23]. Studies of the kinetics of a number of similar chemical reactions, both homogeneous and heterogeneous, show that there is a growing linear dependence between the activation energy $E_{A}$ and the change of the entropy $\Delta S^{\neq}$at the formation of the activated complex from the reagents [24-26]. The establishment of these linear dependences in the studies of the kinetics of similar reactions can be regarded as a proof that the applied kinetic equation describes the specifics of the limiting stage and, furthermore, the kinetic parameters characterizing the process have been correctly calculated.

The purpose of this work is to study the kinetics of non isothermal degradation of rice husks in air or nitrogen atmosphere, polypropylene and tetrafluoroethylene-ethylene copolymer composites, filled with 10 or 20 mass $\%$ rice husks flour or the products of its thermal degradation.

\section{Experimental}

2.1. Theoretical Approach and Mathematical Background. Generally, the kinetic equation and the reaction rate of the chemical reactions can be written as follows $[9,23,27,28]$ :

$$
\frac{\mathrm{d} \alpha}{\mathrm{d} t}=f(\alpha) k(T),
$$

where $\alpha$ is the extent of conversion, $t$ is the time, $f(\alpha)$ is the conversion function, and $k(T)$ is the temperature function, respectively. The temperature dependence of the rate constant $k$ for the reaction is usually described by the Arrhenius equation (2):

$$
k=A \exp \left(-\frac{E_{A}}{R T}\right)
$$

where $A$ is the pre-exponential or frequency factor, $T$ is the absolute temperature, $R$ is the universal gas constant, and $E_{A}$ is the apparent activation energy of the reaction, respectively.
If the studies are carried out under a linear increase of the temperature $\mathrm{d} T / \mathrm{d} t=q=$ const, Equation (1) may be rewritten as follows $[23,27,28]$ :

$$
\frac{\mathrm{d} \alpha}{\mathrm{d} T}=\frac{A}{q} \exp \left(-\frac{E_{A}}{R T}\right) f(\alpha) .
$$

The conversion function $f(\alpha)$ for a solid-state reaction depends on the limiting reaction mechanism and can generally be considered to be as follows:

$$
f(\alpha)=\alpha^{m}(1-\alpha)^{n}[-\ln (1-\alpha)]^{p},
$$

where $m, n$, and $p$ are empirically obtained exponent factors, one of them always being zero [29]. After substitution of (4) in (3), separation of the variables, and integration, the following general equation is obtained $[23,28,29]$ :

$$
\int_{0}^{\alpha} \frac{\mathrm{d} \alpha}{\alpha^{m}(1-\alpha)^{n}[-\ln (1-\alpha)]^{p}}=\frac{A}{q} \int_{0}^{T} \exp \left(-\frac{E_{A}}{R T}\right) \mathrm{d} T .
$$

The solution set of the left hand side integral depends on the explicit expression of the $f(\alpha)$ functions and is denoted as $g(\alpha)$. The algebraic expressions of these functions of the most common reaction mechanisms of solid-state reactions are described elsewhere [9, 23, 30-34]. Coats and Redfern [35] reported that the temperature-containing integral has no exact analytical solution, therefore, they used an approximation. However, the integral does have a primitive analytical solution. Making the substitution $Z=-E /(R T)$ and after a few consecutive integrations by parts, it is possible to reach a recurrent series. Their simplification is quite satisfactory when the thermal energy $(R T)$ is significantly lower than the activation energy $E_{A}$ [36]. This is often the case in the thermal decomposition of solids. One such approximation produces the Coats and Redfern linear equation [35] and may be written as follows:

$$
\ln \frac{g(\alpha)}{T^{2}}=\ln \frac{A R}{q E_{A}}-\frac{E_{A}}{R T} .
$$

This equation is very often used for studying the kinetics of solid-state decomposition reactions [3-5, 31-36].

Tang et al. [37] proposed a new approximate formula for the solution of the Arrhenius temperature integral, which gives the following linear equation:

$$
\begin{aligned}
\ln \left[\frac{g(\alpha)}{T^{1.894661}}\right]= & {\left[\ln \frac{A E_{A}}{q R}+3.63504095-1.894661 \ln E_{A}\right] } \\
& -1.00145033 \frac{E_{A}}{R T} .
\end{aligned}
$$

Wanjun et al. [38] proposed another approximate formula for the Arrhenius temperature integral, which gives the following linear equation:

$$
\ln \left[\frac{g(\alpha)}{T^{2}}\right]=\ln \left[\frac{A R}{q(1.00198882 E+1.87391198 R T)}\right]-\frac{E_{A}}{R T} .
$$


Using (6), (7), or (8) the values of $E_{A}$ and $A$ can be calculated from the slope and cut-off on the ordinate axis. If the correct $g(\alpha)$ function is used, the corresponding plots should give a straight line with high coefficient of the linear regression analysis. It is well known that the formal expressions of the functions $g(\alpha)$ depend on the conversion mechanism and its mathematical model $[23,28,31]$. Both the activation energy and pre-exponential factor are mutually correlated. The correlation can be expressed by the following equation:

$$
\ln A=\ln k_{\text {iso }}+\frac{E_{A}}{R T_{\text {iso }}} .
$$

When the kinetics of reactions of the same type using the correct chosen $g(\alpha)$ function is studied, a linear relationship exists between the corresponding logarithm of the preexponential factors $A$ and the activation energies $E_{A}$ of the reactions studied. This relationship is known as the compensation or isokinetic effect $[2-4,9,23,39-41]$. From the slope and the intercept of the ordinate axis of the straight line drawn, $T_{\text {iso }}$ and $k_{\text {iso, }}$, respectively, can be calculated. At the temperature $T_{\text {iso }}$, all the compounds studied are characterized by the same rate constant, equal to $k_{\text {iso. At }}$ temperatures higher than $T_{\text {iso }}$, the reactions occurring at higher values of $E_{A}$ would have a higher rate (a higher rate constant) than those with lower activation energies. Knowing the values of $E_{A}$ and $A$ for the studied reactions, other kinetic parameters, such as the changes of the Gibbs energy $\Delta G^{\neq}$, enthalpy $\Delta H^{\neq}$, and entropy $\Delta S^{\neq}$for the formation of the activated complex from the reagents, can also be calculated.

According to Eyring's theory for the transition state (activated complex), the frequency factor $A$ in the Arrhenius equation contains an entropy term. The theory uses the following basic equation $[23,31-34,39-41]$ :

$$
k=\frac{\chi k_{\mathrm{B}} T}{h} \exp \left(\frac{\Delta S^{\neq}}{R}\right) \exp \left(-\frac{\Delta H^{\neq}}{R T}\right),
$$

where $\chi$ is the transmission coefficient which accounts for the possibility of the activated complex to decompose with the formation of the end products of the reaction (it is usually equal to unity for monomolecular reactions); $k_{\mathrm{B}}$ is the Boltzmann constant; $h$ is the Plank constant; $\Delta S^{\neq}$and $\Delta H^{\neq}$ are the changes of entropy and enthalpy for the formation of the activated complex from the reagents, respectively. Taking into account the relationship between the activation energy $E_{A}$ and $\Delta H^{\neq}$for the reactions $[2,23,31,39-42]$

$$
E_{A}=\Delta H^{\neq}+n R T,
$$

the following equation is obtained:

$$
k=\frac{\chi e k_{\mathrm{B}} T}{h} \exp \left(\frac{\Delta S^{\neq}}{R}\right) \exp \left(-\frac{E_{A}}{R T}\right),
$$

where $n$ is the molecularity (or order) of the reaction (invariably 1 or 2). In our case, $n$ is equal to unity, $\Delta H^{\neq}$is the change of the enthalpy of activation, and $e=2.7183$ is the Neper number. From the comparison of the Arrhenius equation (2) with (12) it is clear that the frequency factor $A$ is equal to the next term $[23,31,42]$ :

$$
A=\frac{\chi e k_{\mathrm{B}} T}{h} \exp \left(\frac{\Delta S^{\neq}}{R}\right) .
$$

Taking the logarithm from both sides of (13) and rearranging them, we obtain

$$
\Delta S^{\neq}=R \ln \frac{A h}{\chi e k_{\mathrm{B}} T} .
$$

Knowing the value of the frequency factor $A$ and using (14), the value of the change of entropy $\Delta S^{\neq}$for the formation of the activated complex from the reagents can be calculated. Using (11) about the value of the change of enthalpy $\Delta H^{\neq}$and using the well-known Gibbs-Helmholtz thermodynamic relationship [2, 23, 31, 40-42]:

$$
\Delta G^{\neq}=\Delta H^{\neq}-T \Delta S^{\neq},
$$

the change of Gibbs free energy $\Delta G^{\neq}$of the activated complex formation from the reagents can be calculated.

From the value of $\Delta S^{\neq}$obtained, conclusions can be drawn about the degree of arrangement and complexity of the activated complex compared to the initial reagents. The values of $\Delta S^{\neq}$can be either positive or negative, including zero. This is the criterion used to classify the chemical reactions as "fast," "normal," or "slow" [42]. In the cases where the formation of the activated complex from the reagents is not accompanied by significant changes of entropy $\left(\Delta S^{\neq} \approx 0\right)$, the reactions are classified as "normal". For these reactions, the values of their rate constants are close to these calculated using the theory of active impacts. If $\Delta S^{\neq}<0$, as it is in the classic example of the reaction

$$
\mathrm{H}_{2}+\mathrm{I}_{2} \longleftrightarrow 2 \mathrm{HI},
$$

the reaction is classified as "slow." It can be suggested that the activated complex $\left[\mathrm{H}_{2} \mathrm{I}_{2}\right]^{\neq}$formed is more structured or "organized" than the initial molecules, so its formation is accompanied by a decrease of the total entropy of the system [42]. Vice versa, if $\Delta S^{\neq} \gg 0$ for a reaction, it can be suggested that it is a reaction of dissociation of a complex molecule (reagent) to simpler fragments (atoms, radicals, or ions). Such reactions are classified as "fast," for example neutralization of a strong acid with a strong base in solutions. It turns out in a number of cases that a growing linear dependence between $E_{A}$ and the change of entropy $\Delta S^{\neq}$for the formation of the activated complex from the reagents is observed in similar reactions [24-26].

2.2. Materials and Measurements. Raw rice husks (RRHs) from Rice Company, Stara Zagora, Bulgaria were used as a starting material. When dry, they contain about $80 \%$ of organic matter and $20 \%$ inorganic ash. The organic part is composed approximately of $42.8 \% \alpha$-cellulose, $22.5 \%$ lignin, $32.7 \%$ hemicellulose, and about $2 \%$ other organic matter. The inorganic part contains about $95 \%$ silica in amorphous form and has been termed opaline silica. The remaining 5\% 
are some oxides of alkali and alkali earth metals, as well as aluminum and iron oxides [43-45]. RRH was used after thorough washing - three times with tap water followed by two times with deionized water to remove traces of soil, clay, and dust and finally dried at $379 \mathrm{~K}$ for 6 hours. These rice husks were thermally treated in a fluidized bed reactor, shown in Figure 1, at $953 \mathrm{~K}$ in air or nitrogen atmosphere.

The product obtained in air atmosphere contained almost pure ( $>95$ mass $\%$ ) amorphous silica, similar to silica gel, denoted as white rice husks ash (WRHA). The product obtained in nitrogen atmosphere contained about 45 mass $\%$ amorphous carbons, and the rest was amorphous silica, denoted as black rice husks ash (BRHA) [43-45]. The shape of the starting material and the products obtained after the corresponding thermal degradation are presented in Figure 2.

The RRH, BRHA, and WRHA showed in Figure 2 were vigorously ground in an agate mortar and then used as fillers in polyethylene and tetrafluoroethylene-ethylene copolymer composites. The size of the particles was between 20 and $60 \mu \mathrm{m}$.

The thermoplastic polymer polypropylene (PP) "Bulpen" grade 6531, a commercial product of Lukoil-Neftochim, Burgas, Bulgaria with melt index $3-5 \mathrm{~g} / 10 \mathrm{~min}$ $(503 \mathrm{~K} / 2160 \mathrm{~g})$, was used as a matrix for the preparation of the composites. The fillers used were RRH, BRHA, WRHA, and, for comparison, Aerosil A200 (AR), produced by Degussa AG, Germany. Composites with 20 mass $\%$ filler content were prepared by mixing with laboratory mixing rolls at $443 \mathrm{~K}$ for $5 \mathrm{~min}$. The compositions obtained were pressed on an electrically heated hydraulic laboratory press made by PHI, England. The procedure involved softening the sample at $463 \mathrm{~K}$ for $10 \mathrm{~min}$, followed by pressing at $9 \mathrm{MPa}$ for $5 \mathrm{~min}$ at the same temperature and cooling the samples to $313 \mathrm{~K}$ at a rate of $20 \mathrm{~K} \cdot \mathrm{min}^{-1}$.

The TFE-E copolymer used for the experiments was Hostaflon ET-VP 6235 (Hoechst, Germany) with melt index $69.0 \mathrm{~g} / 10 \mathrm{~min}(573 \mathrm{~K}$, and loading $105 \mathrm{~N})$. The compositions were prepared on a Brabender, Germany plastograph by adding 10 mass $\%$ fillers of different nature at $573 \mathrm{~K}$.

All thermogravimetrical measurements were carried out either in air or in nitrogen flow at a rate of $25 \mathrm{~cm}^{3} \cdot \mathrm{min}^{-1}$ under nonisothermal conditions on an MOM-Budapest derivatograph, system F. Paulik-I. Paulik-L. Erdey, which records T, TG, DTG, and DTA curves simultaneously. To minimize and prevent any errors in the measured temperature, we used a platinum crucible and a relatively low heating rate $\left(6 \mathrm{~K} \cdot \mathrm{min}^{-1}\right)$ up to $1173 \mathrm{~K}$. The samples of $50 \mathrm{mg}$ were loaded without pressing into an open crucible. $\alpha$-alumina calcined up to $1373 \mathrm{~K}$ was used as a standard reference material.

\section{Results and Discussion}

The TG and DTG curves of RRH heated in air or nitrogen atmosphere are presented in Figure 3.

According to the data obtained from the thermogravimetric analysis, the thermal decomposition of RRH in air medium occurred in three main stages of mass loss, namely,

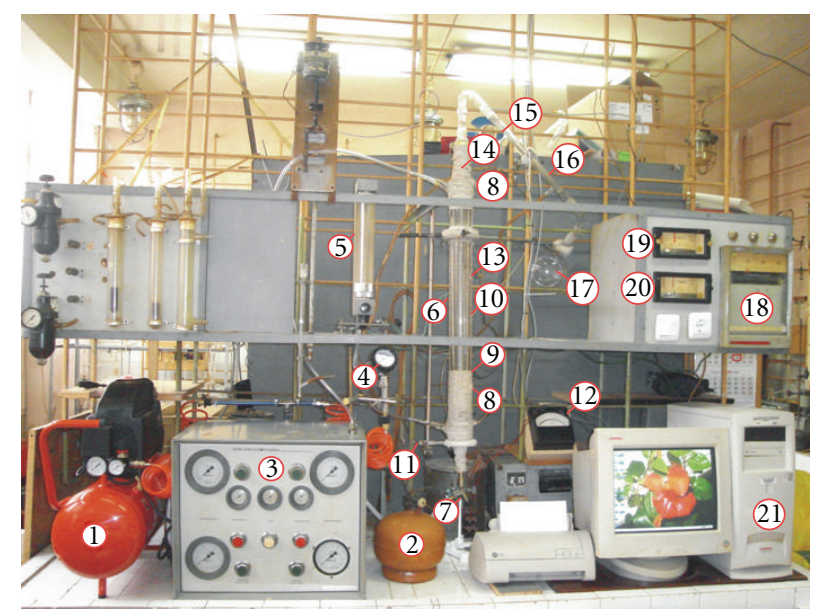

Figure 1: A photograph of the laboratory equipment setup for combustion of raw rice husks in fluidized bed reactor: (1) Black \& Decker air compressor, (2) liquid petroleum gas (LPG) tank, (3) gas distributor, (4) manometer, (5) air Rotameter, (6) quartz fluidized bed reactor, (7) gas burner, (8) asbestos insulator, (9) porous quartz diaphragm, (10) electrical heater, (11) electrical transformer, (12) voltmeter, (13), (14), (15) thermo couples, (16) economizer, (17) separator, (18) temperature recorder, (19), (20) thermo regulators, (21) PC.

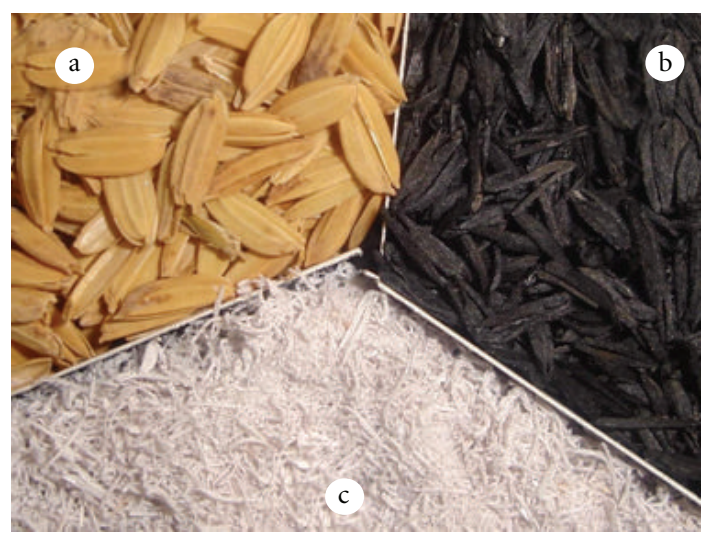

FIGURE 2: Appearance of (a) raw rice husks, (b) black rice husks ash, and (c) white rice husks ash.

removal of moisture (drying), release of organic volatile matters (devolatilization), and oxidation of fixed carbon (slow combustion) [43-45]. As can be seen from Figure 3 (curve A), the mass loss in the first stage took place in the temperature range of 350 to $423 \mathrm{~K}$, and it was accompanied by a small endothermic effect. The mass loss was about $7 \%$ and is associated with the evolution of water adsorbed in the sample and external water bonded by surface tension. The observed features of the thermal decomposition of rice husks can be explained on the basis of the decomposition behaviors of their major constituents: cellulose, hemicellulose, lignin, and ash. According to Stefani et al. [46], hemicellulose degraded first at temperatures between $423 \mathrm{~K}$ and $623 \mathrm{~K}$, then cellulose from 548 to $653 \mathrm{~K}$, and finally lignin from 623 to $823 \mathrm{~K}$. The second and major mass loss of nearly $50 \%$ is 


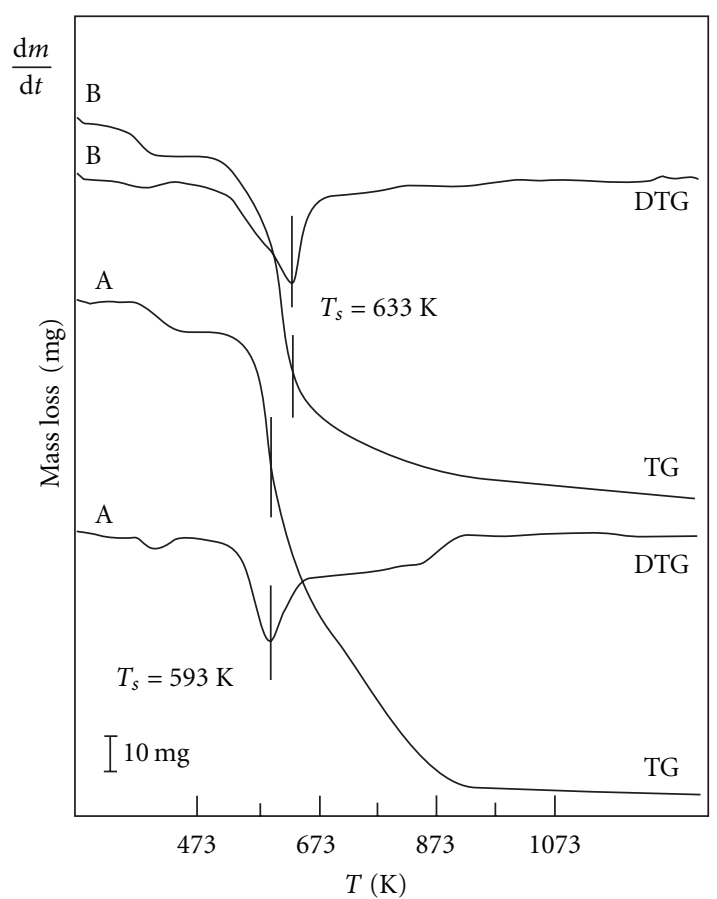

FIGURE 3: TG and DTG curves of RRH: combustion in air flow (a) and pyrolysis in nitrogen flow (b).

attributed to the breakdown of the cellulose constituent to combustible volatiles, water, carbon dioxide, and char. The kinetic mechanism and kinetic parameters of nonisothermal degradation of rice husks in air or nitrogen medium have been described in detail in [43-45]. The smaller kinetic parameters obtained in the third stage compared to these obtained in second stage may be due to the fact that lignin, which has lower decomposition rates than the cellulose and hemicellulose components in rice husks, was condensed to char.

The values of $E_{A}$ and $A$ characterizing the nonisothermal degradation kinetics of rice husks in air or nitrogen atmosphere are calculated on the basis of the corresponding TG curves, (6), (7), and (8). The differences between them are no more than $2.6 \%$. The equation of Ginstling-Brounshtein [44] referring to diffusion-controlled reactions was established as the most appropriate kinetic model. The values of the kinetics parameters, characterizing nonisothermal degradation of the rice husks in air or nitrogen atmosphere calculated according to the Coats-Redfern procedure, are summarized in Table 1 .

As can be seen from the data presented in Table 1, the kinetics of the thermal degradation has two stages. The first one at temperatures lower than $T_{p}$ (peak temperature in DTG curve) is steeper, and the second one at higher temperatures has a smaller slope. For a detailed analysis of the mechanisms of both stages, the values of the activation energy $E_{A}$, frequency factor $A$ in the Arrhenius equation, changes of entropy $\Delta S^{\neq}$, enthalpy $\Delta H^{\neq}$, and Gibbs energy $\Delta G^{\neq}$for the formation of the activated complex from the reagent and the steric factor $P=\exp \left(\Delta S^{\neq} / R\right)$ were calculated [47]. Besides the value of the correlation coefficient of the linear regression analysis $r^{2}$, the second criterion used was $T_{p \text { (calc) }}$. This temperature was calculated from the intersect corresponding straight lines and was compared to the experimentally determined reference temperature $T_{p(\exp ) \text {, }}$ which, in turn, corresponds to the peak temperature in the DTG curve. As can be seen from Table 1, the difference between these two temperatures was less than $4 \mathrm{~K}$. This fact was interpreted as evidence that the Ginstling-Brounshtein equation is the most appropriate mechanism function for describing the studied thermal degradation process, that is, the kinetics of the pyrolysis of rice husks should take into account the diffusion of volatile products from the inside layers of the biomass. The higher values of $E_{A}$ observed at $T<$ $T_{p}$ indicate that the initial release of volatile products occurs under kinetic-diffusion control while the much lower values of $E_{A}$ at $T>T_{p}$ show that the pyrolysis takes place under typical diffusion control. The similar values of activation energy found for the first stages in sample B show that the release of volatile products during burning in air or pyrolysis in nitrogen medium occurs following similar mechanisms.

At the thermal destruction of raw rice husks in air atmosphere there are two main stages: volatilization and burning of released gases and burning of solid carbon containing substances. In the thermal destruction of raw rice husks in nitrogen atmosphere there are the following two stages: release of organic volatile matters without burning and pyrolysis of cellulose, hemicelluloses, and lignin (the main components of raw rice husks) without burning. For this reason, the obtained solid residue in the thermal degradation of raw rice husks in air atmosphere is almost pure silica (>95 mass $\% \mathrm{SiO}_{2}$ ). In the thermal degradation of raw rice husks in nitrogen atmosphere the end solid product contains 45 mass\% amorphous carbons, and the rest is amorphous silica. That is why the values of the activation energy in the two cases are different. In the case of thermal degradation in air atmosphere the process is a complete degradation of the organic matter. In the case of thermal degradation in nitrogen atmosphere the process going on is not so deep and fully destructive, and for this reason the calculated values of activation energy are smaller than in the case of the thermal degradation in air atmosphere.

For monomolecular reactions of decomposition taking place within the solid phase, the values of the frequency factor $A$ of the order of $10^{14} \mathrm{~s}^{-1}$ show that the rotations of the active complex and the reagent do not change during the reaction. At values of $c a \cdot 10^{11} \mathrm{~s}^{-1}$ the reagent can rotate freely while the active complex cannot rotate. The negative values of the change of the entropy mean that the activated complex can be characterized by a much higher "degree of arrangement." Besides, a higher "degree of arrangement" was observed in the second stage (higher negative value of $\Delta S^{\neq}$). The significantly lower than unity values of the steric factor $P$ in the second stage gave enough grounds to classify this stage as "slow." For the first stage, however, the values of $P$ are much closer to unity, so the first stage may be considered as "fast" $[42,43,48]$. The dependence between $\ln A$ and $E_{A}$ was described with the following empiric equation:

$$
\ln A=-10.5048+0.212 E_{A} .
$$


TABLE 1: Kinetic characteristics of the rice husk thermal degradation in air or nitrogen atmosphere.

\begin{tabular}{|c|c|c|c|c|}
\hline \multirow{3}{*}{ Parameter } & \multicolumn{4}{|c|}{ Sample } \\
\hline & \multicolumn{2}{|c|}{ A } & \multicolumn{2}{|c|}{ B } \\
\hline & $T<T_{p}$ & $T>T_{p}$ & $T<T_{p}$ & $T>T_{p}$ \\
\hline$T_{p(\exp .), \mathrm{K}}$ & & & & \\
\hline$T_{p \text { (calc. })}, \mathrm{K}$ & & & & \\
\hline$E_{A}, \mathrm{~kJ} \cdot \mathrm{moL}^{-1}$ & 187 & 23 & 175 & 5 \\
\hline$A, \mathrm{~s}^{-1}$ & $5.83 \times 10^{12}$ & $3.33 \times 10^{-3}$ & $3.01 \times 10^{11}$ & $9.33 \times 10^{-5}$ \\
\hline$k, \mathrm{~s}^{-1}$ & $1.83 \times 10^{-26}$ & $3.01 \times 10^{-5}$ & $1.05 \times 10^{-3}$ & $3.50 \times 10^{-5}$ \\
\hline$\Delta S^{\neq}, \mathrm{J} \cdot \mathrm{moL}^{-1} \cdot \mathrm{K}^{-1}$ & -15 & -306 & -40 & -337 \\
\hline$P$ & 0.171 & $9.88 \times 10^{-17}$ & $8.3 \times 10^{-3}$ & $2.60 \times 10^{-18}$ \\
\hline$\Delta H^{\neq}, \mathrm{KJ} \cdot \mathrm{moL}^{-1}$ & 182 & 18 & 170 & 0.1 \\
\hline$\Delta G^{\neq}, \mathrm{KJ} \cdot \mathrm{moL}^{-1}$ & 191 & 200 & 195 & 213 \\
\hline$r^{2}$ & 0.9899 & 0.9950 & 0.9988 & 0.9822 \\
\hline
\end{tabular}

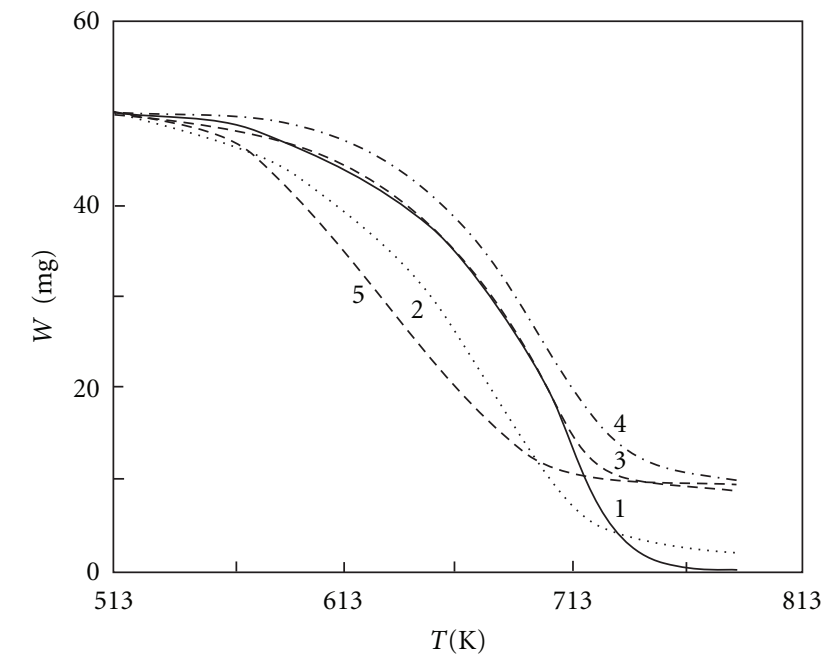

FIGURE 4: TG curves of the thermal degradation in air of pure polypropylene (1) and polypropylene filled with 20 mass $\%$ RRH (2), BRHA (3), WRHA (4) and AR (5).

Based on this equation, the values of the isokinetic temperature $\left(T_{\text {iso }}=567.4 \mathrm{~K}\right)$ and isokinetic rate constant $\left(K_{\text {iso }}=\right.$ $\left.2.73 \times 10^{-5} \mathrm{~s}^{-1}\right)$ were calculated.

The kinetic studies of oxidative thermal degradation of composite materials obtained by filling polypropylene with dispersed fillers of RRH, WRHA, BRHA, and AR have been reported earlier [23]. The thermogravimetric curves of pure polypropylene and polypropylene filled with 20 mass $\%$ fillers of different nature are presented in Figure 4.

As can be seen from Figure 4, the shapes of the TG curves are quite different. Pure polypropylene decomposes without the formation of solid residue (curve 1) while the other samples produce various amounts of solid residue of amorphous $\mathrm{SiO}_{2}$ depending on the nature of the filler used (curves 2 to 5). Besides, the TG curves are shifted to different extents versus that of the pure PP. For example, the TG-curve for polypropylene filled with AR (curve 5) is shifted towards lower temperatures, and the thermal degradation is complete at temperatures up to $713 \mathrm{~K}$, to produce solid residue of about 20 mass\%. It may be concluded therefore that AR as a filler of PP decreases its thermal stability most probably due to the monolayer distribution of PP on the filler and the presence of certain acidic centers on its surface [23]. The TG curve of PP filled with RRH (curve 2) is shifted towards lower temperature versus that of the pure polypropylene because the organic part of the raw rice husks started to decompose at temperature lower than that of pure polypropylene. The TG curve of PP filled with BRHA (curve 3 ) is practically the same as that of pure PP (curve 1). This was attributed to the carbon coating formed on the surface of the amorphous $\mathrm{SiO}_{2}$, which makes it more compatible with the polymer matrix. The kinetics of the oxidative thermal degradation of PP composites can be assessed using the data presented in Table 2.

The highest values of activation energy $E_{A}$ and frequency factor $A$ in the Arrhenius equation were observed for the composites filled with WRHA and AR which have the same chemical content $\left(\mathrm{SiO}_{2}\right)$ and porous structure. The change of Gibbs energy $\Delta G^{\neq}$reflects the total energy increase of the system at the approach of the reagents and the formation of the activated complex. This energy is influenced by two thermodynamic parameters: the changes of enthalpy $\Delta H^{\neq}$and entropy $\Delta S^{\neq}$of the activated complex formation. In all the cases studied, the changes of entropy were negative, which showed that the activated complex is a more "organized" structure than the initial reagents. There is obviously a relationship between the values of $E_{A}, \ln A$, and $\Delta S^{\neq}$: higher values of $\ln A$ correspond to higher values of $E_{A}$ and less negative values of $\Delta S^{\neq}$[47]. The isokinetic rate constant $k_{\text {iso }}$ was calculated to be equal to $2.68 \times 10^{-4} \mathrm{~s}^{-1}$ and isokinetic temperature $T_{\text {iso }}=574.4 \mathrm{~K}$.

The TG curves of the initial TFE-E copolymer and its composites, contained 10 mass\% WRHA, BRHA, or Aerosil Degussa A200 [49] taken at heating rate of $6 \mathrm{~K} \cdot \mathrm{min}^{-1}$ are presented in Figure 5.

It can be seen from Figure 5 that the thermal degradation of the initial TFE-E copolymer (curve 1) started at the highest temperature and the corresponding TG curve for the sample filled with 10 mass\% BRHA (curve 2) is close to it, while the TG curves of the copolymers filled with 10 mass\% WRHA 
TABLe 2: Kinetic parameters of nonisothermal degradation in air of fillers of different nature (20 mass\%) PP [23].

\begin{tabular}{|c|c|c|c|c|c|}
\hline \multirow{2}{*}{ Parameter } & \multicolumn{5}{|c|}{ Sample } \\
\hline & PP & PP-RRH & PP-BRHA & PP-WRHA & PP-AR \\
\hline$E_{A}, \mathrm{~kJ} \cdot \mathrm{moL}^{-1}$ & 67 & 72 & 64 & 109 & 113 \\
\hline$A, \mathrm{~s}^{-1}$ & $2.42 \times 10^{2}$ & $1.25 \times 10^{4}$ & $1.56 \times 10^{3}$ & $8.15 \times 10^{5}$ & $1.06 \times 10^{7}$ \\
\hline$\Delta S^{\neq}, \mathrm{J} \cdot \mathrm{moL}^{-1} \cdot \mathrm{K}^{-1}$ & -215 & -201 & -218 & -147 & -125 \\
\hline$\Delta H^{\neq}, \mathrm{kJ} \cdot \mathrm{moL}^{-1}$ & 61 & 66 & 58 & 103 & 107 \\
\hline$\Delta G^{\neq}, \mathrm{kJ} \cdot \mathrm{moL}^{-1}$ & 216 & 205 & 212 & 208 & 187 \\
\hline
\end{tabular}

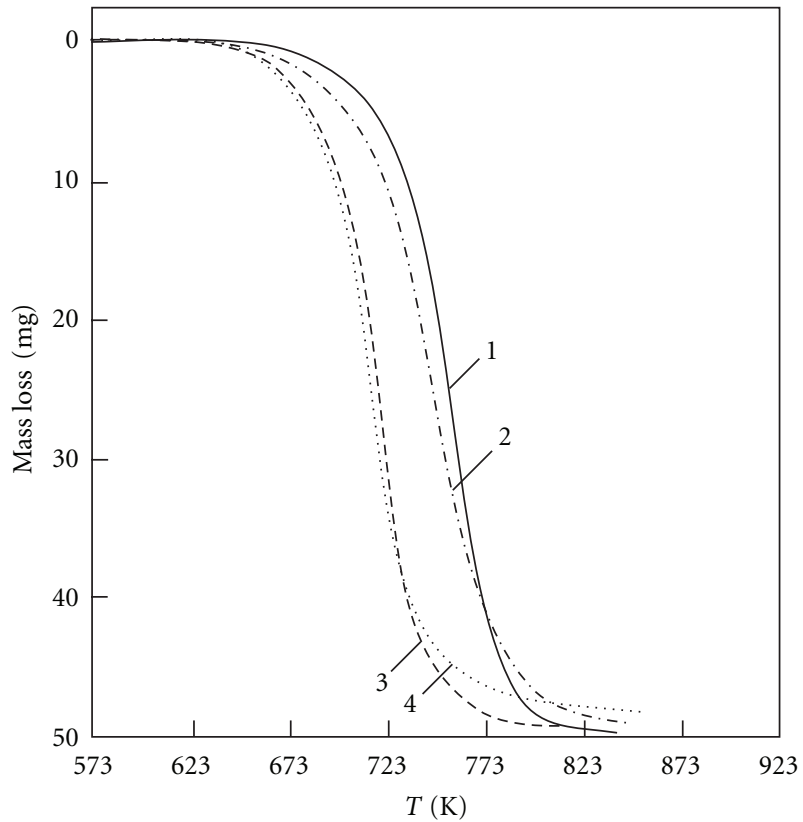

FIGURE 5: TG curves for thermal degradation in air of: TFE-E copolymer (1), TFE-E + BRHA (2), TFE-E + WRHA (3) and TFE-E $+\mathrm{AR}(4)$.

or AR (curves 3 and 4) are shifted to lower temperatures and practically coincide. This indicates that the fillers used decreased the thermal stability of the initial copolymer to different extents.

The weak influence of BRHA can be explained by the fact that its surface was carbonized, which makes it more hydrophobic and more compatible with the initial copolymer. Vice versa, WRHA and AR, which are practically pure amorphous $\mathrm{SiO}_{2}$, have a hydrophilic behavior and lower compatibility with the TFE-E copolymer. Besides, these fillers probably reduced its due to the surface $\mathrm{Si}-\mathrm{OH}$ groups. It is well known from the literature $[23,48]$ that some solid acids, such as amorphous alumina, silica-alumina, clays, natural and synthetic zeolites, which have Brnsted or Lewis strong acid centers, can promote polymer chain cracking. Since the shapes of the TG curves shown in Figure 5 are different, it could be expected that the values of the activation energies $E_{A}$ and the frequency factor $A$ in the Arrhenius equation characterizing the thermooxidative degradation kinetics of the studied composites would be different. According to the TG data, the oxidative nonisothermal degradation of the composites studied occurred in two stages. The values of the obtained kinetics parameters for all the samples studied are presented in Table 3.

As can be seen from Table 3, for both stages the initial TFE-E copolymer had the highest values of $E$, and $A$ and the sample filled with 10 mass $\%$ BRHA had the lowest. Supposedly, it was due to the fact that this filler contained a significant amount of porous amorphous carbon which burned under heating in air and facilitated the thermooxidative degradation of the TFE-E copolymer. The close values of $E$ and $A$ for both stages of the samples filled with WRHA or AR were considered to be due to their similar nature. The fact that they were lower than these of the initial TFEE copolymer showed that the surface acidic centers of these fillers play a catalytic role in the thermooxidative degradation of the TFE-E copolymer in air. The big differences between the values of the pre-exponential factor $A$ for both stages are very interesting. In the cases when the values of $A$ are between $10^{10}$ and $10^{12} \mathrm{~s}^{-1}$, the complex was probably highly restricted in rotation compared to the initial reagent [47]. For the unimolecular case, the complex is supposed to expand by size and, hence, interact more intensely with its neighbors. At values of $A$ less than $10^{6} \mathrm{~s}^{-1}$, the reagent is considered to be in equilibrium with the surface adsorption layer to become a product after passing through the activated complex. Such a situation can be observed during the second stage of decomposition since, in this case, significant amounts of monomers which are the final products of the degradation are adsorbed on the filler surface. Since the frequency factor $A$ contains an entropy term $\left(\exp \Delta S^{\neq} / R\right)$, the values of $A$ reflect in a specific way on the change of the entropy $\Delta S^{\neq}$for the formation of the activated complex from the reagents. For example, the negative values of $\Delta S^{\neq}$obtained (see Table 3) would indicate that the formation of the activated complex is connected with a decrease of entropy, that is, the activated complex is a "more organized" structure compared to the initial substance and such reactions are classified as "slow." On the basis of the linear relationship obtained between $\ln A$ and $E_{A}$, the average values of $k_{\text {iso }}=4.17 \times$ $10^{-4} \mathrm{~s}^{-1}$ and $T_{\text {iso }}=664.8 \mathrm{~K}$ were calculated, respectively.

The graphical representation of the dependence between the values of $E_{A}$ and $\Delta S^{\neq}$for all the polymer composites studied clearly shows the good linear relationship between them Figure (6).

It can be seen from Figure 6 that the dependence between $E_{A}$ and $\Delta S^{\neq}$was linear for all the three systems studied. As it has been reported $[23,43,48]$, the existence of unambiguous linear dependence between $\ln A$ and $E_{A}$, that is, a compensation effect, can be interpreted as a proof that 
TABLE 3: Kinetic parameters of thermooxidative degradation in air of TFE-E and composites filled with 10 mass $\%$ BRHA, WRHA, or AR [49].

\begin{tabular}{lcccccc}
\hline Sample & \multicolumn{3}{c}{ Ist stage } & \multicolumn{2}{c}{ Ind stage } \\
& $E, \mathrm{~kJ} \cdot \mathrm{moL}^{-1}$ & $A, \mathrm{~s}^{-1}$ & $\Delta S^{\neq}, \mathrm{J} \cdot \mathrm{moL}^{-1} \cdot \mathrm{K}^{-1}$ & $E, \mathrm{~kJ} \cdot \mathrm{moL}^{-1}$ & $A, \mathrm{~s}^{-1}$ & $\Delta S^{\neq}, \mathrm{J} \cdot \mathrm{moL}^{-1} \cdot \mathrm{K}^{-1}$ \\
\hline TFE-E & 182 & $2.78 \times 10^{10}$ & -60 & 97 & $2.30 \times 10^{4}$ & -178 \\
TFE-E+10\% BRHA & 145 & $4.17 \times 10^{7}$ & -114 & 33 & 0.126 & -281 \\
TFE-E + 10\% WRHA & 163 & $1.30 \times 10^{9}$ & -86 & 56 & $1.59 \times 10^{1}$ & -238 \\
TFE-E + 10\% AR & 161 & $1.03 \times 10^{9}$ & -88 & 51 & 7.07 & -245 \\
\hline
\end{tabular}

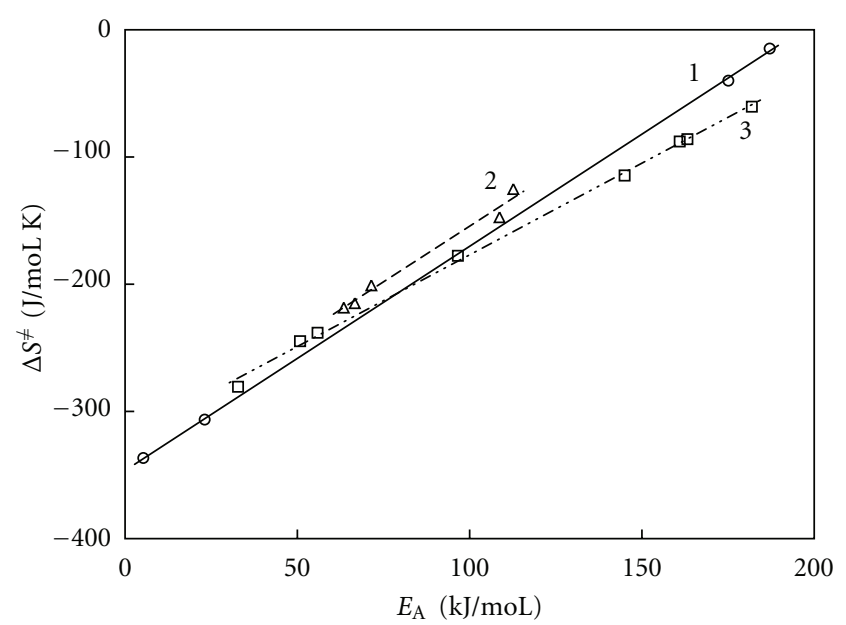

FIGURE 6: Plot of $\Delta S^{\neq}$versus $E_{A}$ for thermal degradation of RRH in air or nitrogen medium (1), PP composites (2), and TFE-E copolymer composites (3), filled with different fillers.

the mechanistic kinetic equations used adequately reflect the specifics of the limiting stage, and, furthermore, the kinetic parameters characterizing these processes were correctly calculated. The existence of a linear dependence between $\Delta S^{\neq}$ and $E_{A}$ provides a solid basis for seeking a direct relationship between them, that is, the values of the activation energies of the similar destruction processes of the polymer materials studied exert a certain effect on the values of the change of entropy for the formation of the corresponding activated complexes from the initial reagents. Besides, it can be seen that the higher the value of $E_{A}$ the lower the value of the corresponding $\Delta S^{\neq}$, with $\Delta S^{\neq}$values remaining negative. According to the Arrhenius theory, an effective interaction between reagents requires a certain amount of energy, denoted as activation energy $E_{A}$. Nevertheless, the formation of the activated complex is accompanied by different changes in the reagents structure, which is accounted for by the frequency factor $A$ or, to be precise, the entropy term it contains $\left(\exp \Delta S^{\neq} / R\right)$. The latter can be either smaller or bigger than unity, including unity, which is determined by the value of $\Delta S^{\neq}$. The relationships observed can be interpreted first as a proof that the activated complexes have a "more organized" structure compared to the initial reagents and, second, the rearrangement taking place is accompanied by a decrease of the system entropy. This is explained by the fact that the degrees of freedom of the activated complex are less than these of the initial reagents. Since the activated complex exists for about $5 \times 10^{-13} \mathrm{~s}$, it can be regarded as quasimolecule differing from the usual one by the lack of vibration on the reaction path. This vibration is replaced by the translational movement of the activated complex on the reaction path and the forming of reaction products [41].

Figure 6 showed also that the slopes and the intercepts of the three straight lines were almost the same. This was considered to be due to the great similarity between the kinetic mechanisms of the degradation processes taking place in the polymer materials studied. It was also confirmed by the close values of $K_{\text {iso }}$ and $T_{\text {iso }}$ calculated for the different composites. Therefore, it may be suggested that the differences in the values of $E_{A}$ and $\Delta S^{\neq}$reflect the specific features of the individual systems while the close values of the slopes and intercepts of the three straight lines appear to be a group characteristic of processes of the same type.

\section{Conclusions}

Using three different calculation procedures, the values of $E_{A}$ and the frequency factor $A$ in the Arrhenius equation are calculated for nonisothermal degradation of rice husks in air or nitrogen atmosphere and polypropylene and tetrafluoroethylene-ethylene copolymer filled with different quantities of rice husks flour or the products of their thermal degradation. For the studied samples a linear dependence was found between $\ln A$ and $E_{A}$, known as the kinetic compensation effect or theta rule. A linear relationship was also established between $E_{A}$ and the change of the entropy $\Delta S^{\neq}$for the formation of the activated complex from the reagents. These dependences are related to the assumption that the kinetic mechanisms of thermal degradation of the composites studied were identical. The negative values of $\Delta S^{\neq}$obtained showed that the activated complex is a "more organized" structure than the initial reactants and that these reactions may be classified as a "slow." The observed differences in the values of the kinetics parameters of nonisothermal degradation of the studied samples are results of the nature of the used fillers. The existence of different quantities of silanol and siloxan groups on the surface of amorphous $\mathrm{SiO}_{2}$ affects the thermal stability of the studied composite materials. Because BRHA has a hydrophobic surface, it is more compatible with the polymer matrix and may be successfully used as a filler of polymer composites.

In conclusion, it may be stated that the products of the thermal degradation of rice husks in a fluidized bed reactor may successfully replace the more expensive synthetic fillers 
in the production of different polymer composites. These polymer composites can lead to the futuristic "organicinorganic hybrid materials" with specific properties.

\section{References}

[1] M. E. Brown and A. K. Galwey, "The significance of "compensation effects" appearing in data published in "computational aspects of kinetic analysis": ICTAC project," Thermochimica Acta, vol. 387, no. 2, pp. 173-183, 2002.

[2] R. Bigda and A. Mianowski, "Influence of heating rate on kinetic quantities of solid phase thermal decomposition," Journal of Thermal Analysis and Calorimetry, vol. 84, no. 2, pp. 453-465, 2006.

[3] B. Jankovich and S. Mentus, "Model-fitting and model-free analysis of thermal decomposition of palladium acetylacetonate $\left[\mathrm{Pd}(\mathrm{acac})_{2}\right]$," Journal of Thermal Analysis and Calorimetry, vol. 94, no. 2, pp. 395-403, 2008.

[4] A. Rotaru, A. Moanta, P. Rotaru, and E. Segal, "Tdecomposition kinetics of some aromatic azomonoethers," Journal of Thermal Analysis and Calorimetry, vol. 95, no. 1, pp. 161-166, 2009.

[5] R. López-Fonseca, I. Landa, M. A. Gutiérrez-Ortiz, and J. R. González-Velasco, "Non-isothermal analysis of the kinetic of the combustion of carbonaceous materials," Journal of Thermal Analysis and Calorimetry, vol. 80, no. 1, pp. 65-69, 2005.

[6] J. Norwisz and T. Musielak, "Compensation law again," Journal of Thermal Analysis and Calorimetry, vol. 88, no. 3, pp. 751-755, 2007.

[7] D. S. Dias, M. S. Crespi, and C. A. Ribeiro, "Non-isothermal decomposition kinetics of the interaction of poly(ethylene terephthalate) with alkyd varnish," Journal of Thermal Analysis and Calorimetry, vol. 94, no. 2, pp. 539-543, 2008.

[8] B. Janković, L. Kolar-Anić, I. Smičiklas, and S. Dimović, "The non-isothermal thermogravimetric tests of animal bones combustion. Part. I. Kinetic analysis," Thermochimica Acta, vol. 495, no. 1-2, pp. 129-138, 2009.

[9] "Reactions in the solid state," in Comprehensive Chemical Kinetic, M. E. Brown, D. Dollimore, and A. K. Galwey, Eds., vol. 22, Elsevier, Amsterdam, The Netherlands, 1th edition, 1980.

[10] P. D. Garn, "The kinetic compensation effect," in Proceedings of the 4th International Conference on Thermal Analysis (ICTA '74), I. Buzas, Ed., vol. 1 of Thermal Analysis, pp. 25-31, Budapest, Hungary', 1974.

[11] A. V. Nikolaev, V. A. Logvinenko, V. M. Gorbatchov, and L. I. Myachina, "Thermal analysis," in Proceedings of the 4th International Conference on Thermal Analysis (ICTA '74), vol. 1, pp. 47-55, Budapest, Hungary, 1974.

[12] T. Zmijewski and J. Pysiak, "Thermal analysis," in Proceedings of the 4th International Conference on Thermal Analysis (ICTA '74), vol. 1, pp. 205-213, Budapest, Hungary, 1974.

[13] D. Dollimore and T. Taylor, "The appearance of a compensation effect in the rising temperature decomposition of a series of pure carbonates," Thermochimica Acta, vol. 40, no. 2, pp. 297-304, 1980.

[14] N. Koga and H. Tanaka, "Significance of kinetic compensation effect in the thermal decomposition of a solid," Thermochimica Acta, vol. 135, pp. 79-84, 1988.

[15] N. Koga and H. Tanaka, "Conventional kinetic analysis of the thermogravimetric curves for the thermal decomposition of a solid," Thermochimica Acta, vol. 183, pp. 125-136, 1991.
[16] P. Budrugeac, "The kinetics of thermal degradation of polymers exhibiting a compensation effect and a linear variation of activation energy with degree of degradation," Polymer Degradation and Stability, vol. 38, no. 3, pp. 229-233, 1992.

[17] A. Corma, F. Llopis, J. Monton, and S. Weller, "On thecompensation effect in acid-base catalyzed reactions on zeolites," Journal of Catalysis, vol. 142, no. 1, pp. 97-109, 1993.

[18] Z. Adonyi and G. Kórösi, "Experimental study of nonisothermal kinetic equations and compensation effect," Thermochimica Acta, vol. 60, no. 1, pp. 23-45, 1993.

[19] N. Koga, "A review of the mutual dependence of Arrhenius parameters evaluated by the thermoanalytical study of solidstate reactions: the kinetic compensation effect," Thermochimica Acta, vol. 244, pp. 1-20, 1994.

[20] J. Zsakó, "Compensation effect in heterogeneous non-isothermal kinetics," Journal of Thermal Analysis, vol. 47, no. 6, pp. 1679-1690, 1996.

[21] A. K. Galwey, "Compensation behaviour recognized in literature reports of selected heterogeneous catalytic reactions: aspects of the comparative analyses and significance of published kinetic data," Thermochimica Acta, vol. 294, no. 2, pp. 205-219, 1997.

[22] A. Ruvolo-Filho and P. Curti, "Chemical kinetic model and thermodynamiccompensation effectof alkaline hydrolysis in waste poly(ethylene terephtalate) in non-aqueous ethylene glycol solution," Industrial and Engineering Chemistry Research, vol. 45, no. 24, pp. 7985-7996, 2006.

[23] S. C. H. Turmanova, S. D. Genieva, A. S. Dimitrova, and L. T. Vlaev, "Non-isothermal degradation kinetics of filled with rise husk ash polypropene composites," Express Polymer Letters, vol. 2, no. 2, pp. 133-146, 2008.

[24] I. Horvath, "Kinetics and compensation effect in kaolinite dehydroxylation," Thermochimica Acta, vol. 85, pp. 193-198, 1985.

[25] L. Singh and S. Mitra, "Thermal investigation and stereochemical studies of some cyclic diamine complexes of nickel(II), zinc(II) and cadmium(II) in the solid state. Part II," Thermochimica Acta, vol. 138, no. 2, pp. 285-301, 1989.

[26] S. Rudra, H. Singh, S. Basu, and U. Shivhare, "Enthalpy entropy compensation during thermal degradation of chlorophyll in mint and coriander puree," Journal of Food Engineering, vol. 86, no. 3, pp. 379-387, 2008.

[27] P. Budrugeac and E. Segal, "Some methodological problems concerning nonisothermal kinetic analysis of heterogeneous solid-gas reactions," International Journal of Chemical Kinetics, vol. 33, no. 10, pp. 564-573, 2001.

[28] S. D. Genieva, L. T. Vlaev, and A. N. Atanassov, "Study of the thermooxidative degradation kinetics of poly(tetrafluoroethene) using iso-conversional calculation procedure," Journal of Thermal Analysis and Calorimetry, vol. 99, no. 2, pp. 551-561, 2010.

[29] J. Šestak and G. Berggren, "Study of the kinetics of the mechanism of solid-state reactions at increasing temperatures," Thermochimica Acta, vol. 3, no. 1, pp. 1-12, 1971.

[30] L. Minying, G. Lijun, Z. Qingxiang, W. Yudong, Y. Xiaojuan, and C. Shaokui, "Thermal degradation process and kinetics of poly(dodecamethyleneisophthalamide)," Chemical Journal on Internet, vol. 5, no. 6, pp. 1-13, 2003.

[31] L. T. Vlaev, M. M. Nikolova, and G. G. Gospodinov, "Study on the kinetics of non-isothermal dehydration of alkaline earth metal selenites," Chemical Monthly, vol. 136, no. 9, pp. 15531566, 2005. 
[32] P. Budrugeac, "The Kissinger law and the IKP method for evaluating the non-isothermal kinetic parameters," Journal of Thermal Analysis and Calorimetry, vol. 89, no. 1, pp. 143-151, 2007.

[33] F. Carrasco, D. Dionisi, A. Martinelli, and M. Majone, "Thermal stability of polyhydroxyalkanoates," Journal of Applied Polymer Science, vol. 100, no. 3, pp. 2111-2121, 2006.

[34] K. Chrissafis, "Kinetics of thermal degradation of polymers: complementary use of isoconversional and model-fitting methods," Journal of Thermal Analysis and Calorimetry, vol. 95, no. 1, pp. 273-283, 2009.

[35] A. W. Coats and J. P. Redfern, "Kinetic parameters from thermogravimetric data," Nature, vol. 201, no. 4914, pp. 68-69, 1964.

[36] F. Carrasco, "The evaluation of kinetic parameters from thermogravimetric data: comparison between established methods and the general analytical equation," Thermochimica Acta, vol. 213, pp. 115-134, 1993.

[37] W. Tang, Y. Liu, H. Zhang, and C. Wang, "New approximate formula for Arrhenius temperature integral," Thermochimica Acta, vol. 408, no. 1-2, pp. 39-43, 2003.

[38] T. Wanjun, L. Yuwen, Z. Hen, W. Zhiyong, and W. Cunxin, "New temperature integral approximate formula for nonisothermal kinetic analysis," Journal of Thermal Analysis and Calorimetry, vol. 74, no. 1, pp. 309-315, 2003.

[39] N. Pop, G. Vlase, T. Vlase, N. Doca, A. Mogos, and A. Ioitescu, "Compensation effect as a consequence of vibrational energy transfer in homogeneous and isotropic heat field," Journal of Thermal Analysis and Calorimetry, vol. 92, no. 1, pp. 313-317, 2008.

[40] F. Tûdos and P. David, "Comments on the eyring interpretation of the compensation effect," Polymer Degradation and Stability, vol. 50, no. 2, pp. 159-161, 1995.

[41] M. Olszak-Humienik, "Dependence of thermal decomposition rate constant on temperature of reaction," Journal of Thermal Analysis and Calorimetry, vol. 65, no. 2, pp. 515-518, 2001.

[42] D. V. Sokol'skii and V. A. Druz, Introduction in Theory of Heterogeneous Catalysis, Vyshaya Shkola, Moscow, Russia, 1981.

[43] S. D. Genieva, S. C. H. Turmanova, A. S. Dimitrova, and L. T. Vlaev, "Characterization of rice husks and the products of its thermal degradation in air or nitrogen atmosphere," Journal of Thermal Analysis and Calorimetry, vol. 93, no. 2, pp. 387-396, 2008.

[44] L. T. Vlaev, I. G. Markovska, and L. A. Lyubchev, "Nonisothermal kinetics of pyrolysis of rice husk," Thermochimica Acta, vol. 406, no. 1-2, pp. 1-7, 2003.

[45] I. G. Markovska and L. A. Lyubchev, "A study on the thermal destruction of rice husk in air and nitrogen atmosphere," Journal of Thermal Analysis and Calorimetry, vol. 89, no. 3, pp. 809-814, 2007.

[46] P. Stefani, D. Garcia, J. Lopez, and A. Jimenez, “Thermogravimetric analysis of composites obtained from sintering of rice husk-scrap tire mixtures," Journal of Thermal Analysis and Calorimetry, vol. 81, no. 2, pp. 315-320, 2005.

[47] A. Sharma and T. Rajeswara Rao, "Kinetics of pyrolysis of rice husk," Bioresource Technology, vol. 67, no. 1, pp. 53-59, 1999.

[48] L. T. Vlaev, I. G. Markovska, and L. A. Lyubchev, "Kinetics compensation effect at thermal degradation of rice husk," Oxidation Communications, vol. 27, no. 2, pp. 444-452, 2004.
[49] A. Atanassov, S. Genieva, and L. Vlaev, "Study on the thermooxidative degradation kinetics of tetrafluoroethyleneethylene copolymer filled with rice husks ash," PolymerPlastics Technology and Engineering, vol. 49, no. 6, pp. 541-554, 2010. 

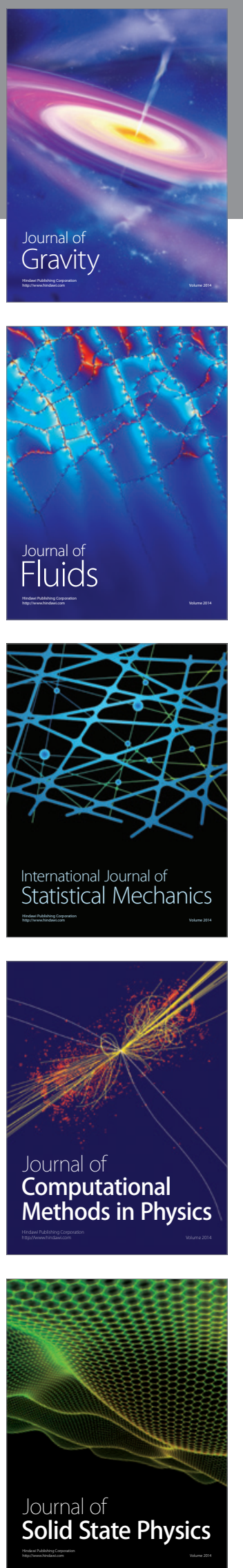

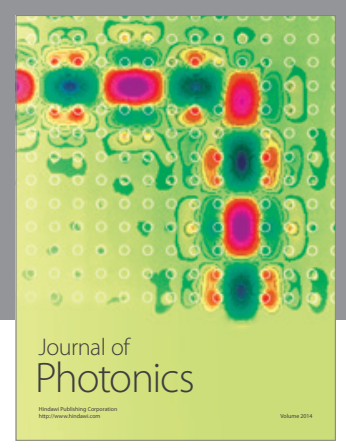

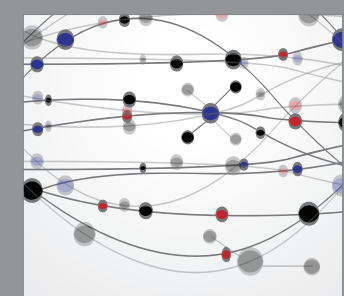

The Scientific World Journal
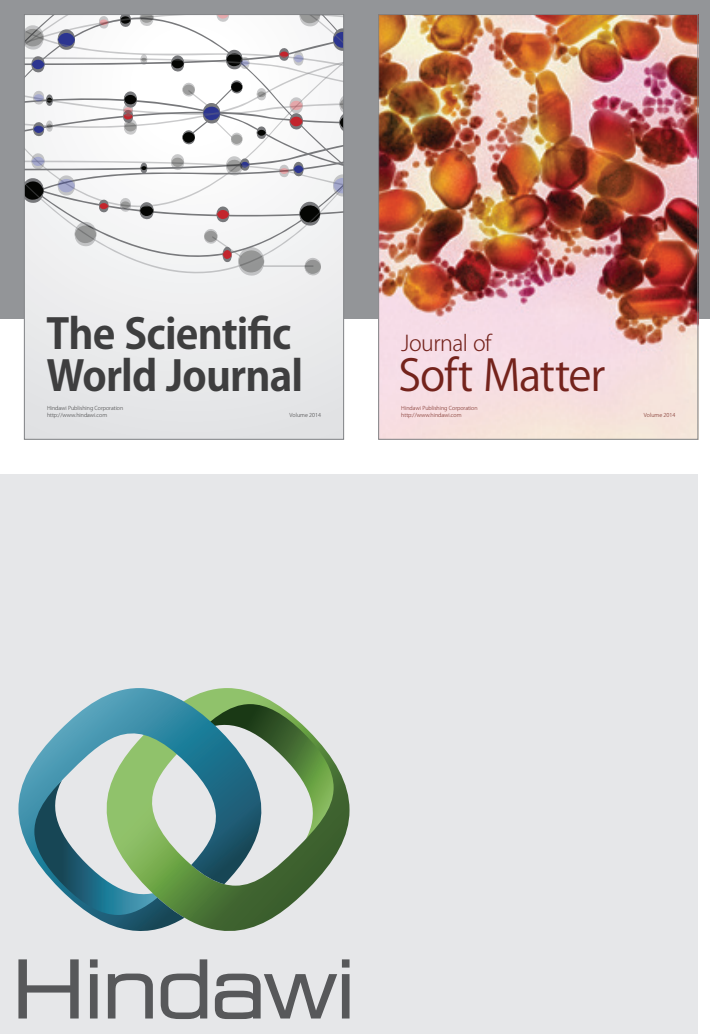

Submit your manuscripts at

http://www.hindawi.com
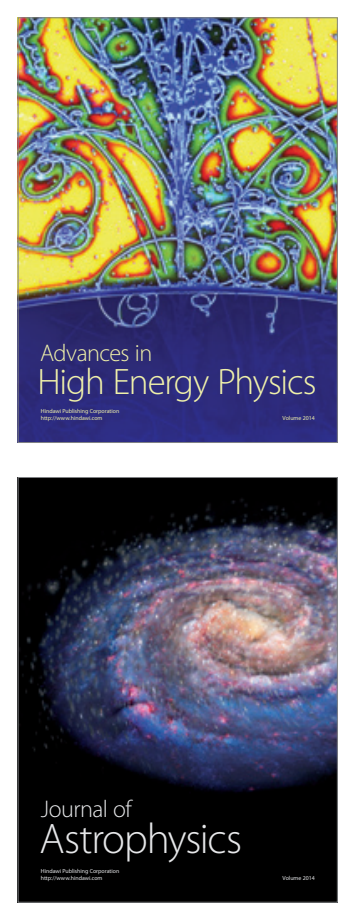
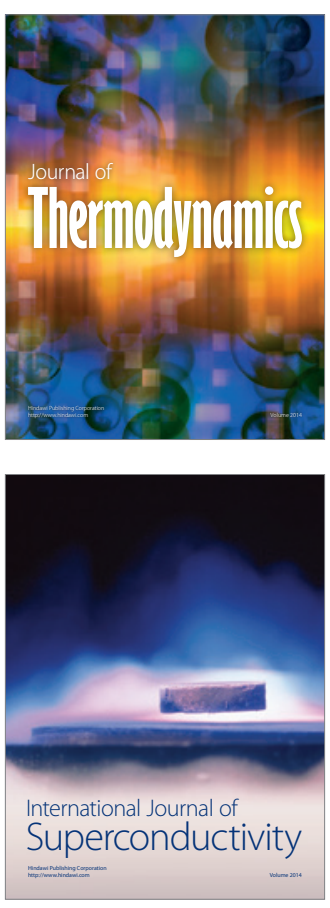
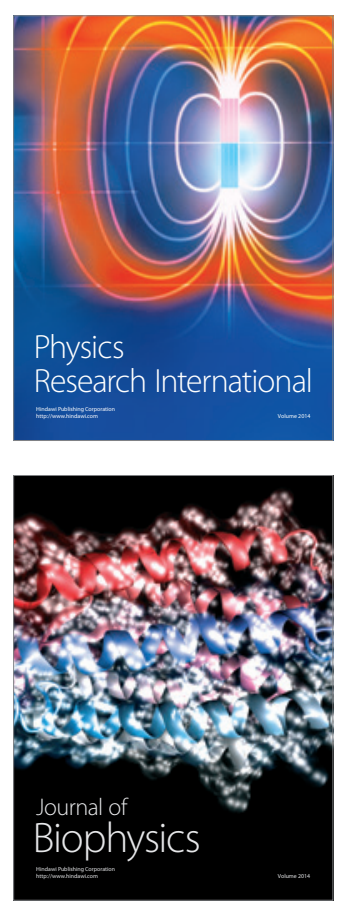
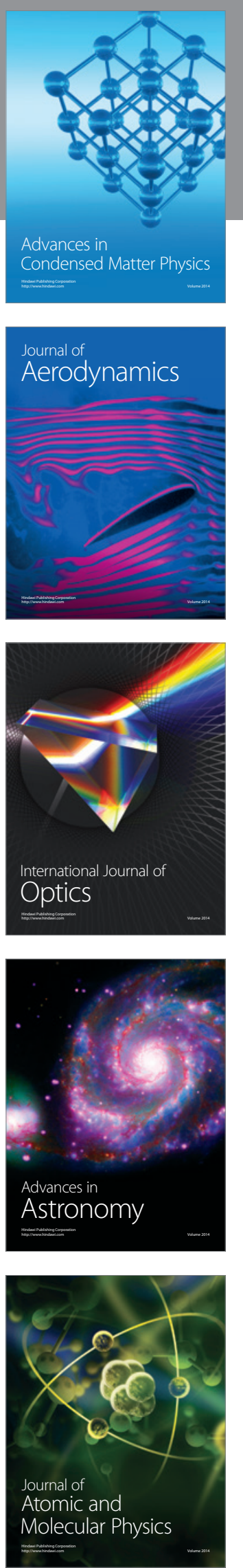